\title{
Active Control for Multinode Unbalanced Vibration of Flexible Spindle Rotor System with Active Magnetic Bearing
}

\author{
Xiaoli Qiao and Guojun Hu \\ Shaoxing University Yuanpei College, Shaoxing 312000, China \\ Correspondence should be addressed to Guojun Hu; 514914515@qq.com
}

Received 3 March 2017; Accepted 24 April 2017; Published 6 June 2017

Academic Editor: Aly-Mousaad Aly

Copyright ( 2017 Xiaoli Qiao and Guojun Hu. This is an open access article distributed under the Creative Commons Attribution License, which permits unrestricted use, distribution, and reproduction in any medium, provided the original work is properly cited.

The unbalanced vibration of the spindle rotor system in high-speed cutting processes not only seriously affects the surface quality of the machined products, but also greatly reduces the service life of the electric spindle. However, since the unbalanced vibration is often distributed on different node positions, the multinode unbalanced vibration greatly exacerbates the difficulty of vibration control. Based on the traditional influence coefficient method for controlling the vibration of a flexible rotor, the optimal influence coefficient control method with weights for multinode unbalanced vibration of flexible electric spindle rotors is proposed. The unbalanced vibration of all nodes on the whole spindle rotor is used as the control objective function to achieve optimal control. The simulation results show that the method has an obvious control effect on multinode unbalanced vibration.

\section{Introductions}

Maglev support technology is one of the world's most recognized high-tech innovations. It can be used as a support technology for high-speed machine electric spindles in high-end CNC machine tools. With the increase of the spindle rotational speed, the spindle will show flexibility characteristics when the speed of the spindle rotor exceeds its critical speed. When a flexible spindle rotor is running at the first or higher critical speed, the different vibration mode determines the response characteristics of the spindle. Therefore, a real-time active control technique is required for the unbalanced vibration control of the flexible spindle rotor system. According to the vibration mode characteristics of the flexible spindle rotor, the control force is changed in real time, so that the vibration suppression of the rotor is optimized.

The conventional flexible rotor unbalanced vibration control method can be divided into two categories: modal method and influence coefficient method [1]. The biggest advantage of the coefficient method is that it does not depend on the mathematical model of the control object, which is independent of the modal characteristics of the rotor, and directly achieves vibration control based on the relationships between the correction force and rotor node displacement. So, the influence coefficient method is one of the most promising methods in the field of unbalanced vibration control of flexible rotors. There are many studies on vibration control by use of the influence coefficient method. Tseng et al. [2] proposed a real-time dynamic balance scheme by the influence coefficient method. Li et al. [3] used the influence coefficient method to carry out the first two-order flexible modes of the rotor, respectively. Kim and Lee [4] recognized the sensor runout with the influence coefficient method without applying additional sensors and equipment and applied the influence coefficient method to the harmonic vibration suppression of the flexible rotor. Kang et al. [5] used an automatic balance method based on the influence coefficient method. The influence coefficient balancing equations, with suitable constraints on the level of the residual vibrations and the magnitude of correction weights, were cast in linear matrix inequality (LMI) forms and solved with the numerical algorithms developed in convex optimization theory by Untaroiu et al. [6]. Yu et al. [7] used the active magnetic bearing as the actuator, which detected the transfer function model to obtain the influence coefficient matrix to achieve the vibration suppression of the flexible spindle rotor. Knospe [8] proposed a flexible rotor unbalance control 


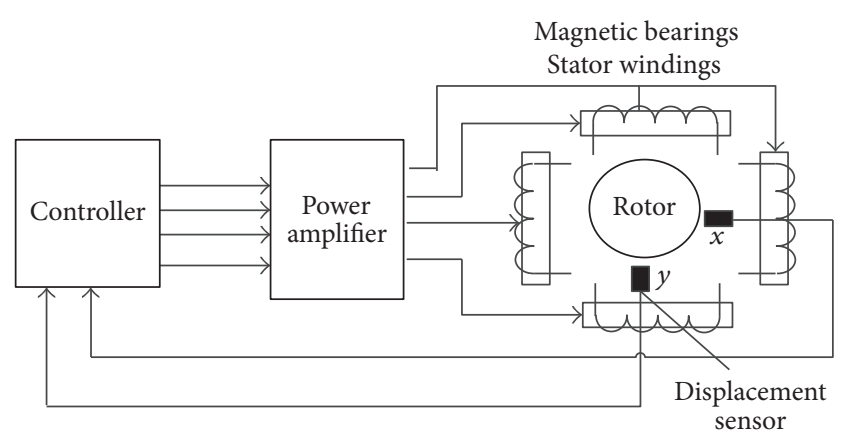

FIgURE 1: The working principle of an active magnetic bearing.

method based on the influence coefficient method. Lee et al. [9] proposed an active balancing method for rotating machinery using the influence coefficient method. Kang et al. [10] studied the optimal balancing of flexible rotors by minimizing the condition number of influence coefficients, and so on.

The flexible spindle vibration control method with active magnetic bearing is characterized in real time and online. According to the real-time situation of the flexible spindle vibration, the active magnetic bearing can adjust the electromagnetic correction force online to make the vibration control for the flexible spindle rotor achieve the best effect. So, the influence coefficient method with active magnetic bearing is the most widely used method for unbalanced control in flexible spindle rotors.

The traditional influence coefficient method for controlling flexible spindle rotor vibration needs to calculate the electromagnetic force by solving the multivariate system, which causes the number of vibration suppression nodes to be not more than the number of excitation nodes of the active magnetic bearing. In order to solve the above problem, the paper puts forward the optimal influence coefficient control method with weights for multinode unbalanced vibrations of flexible electric spindle rotors, which uses all the unbalanced vibration nodes of the whole spindle as the control objective function to achieve the optimal control of multinode unbalanced vibration.

\section{Principle of Active Magnetic Bearing}

A simplified active magnetic bearing control schematic diagram is shown in Figure 1; the rotor is free to be suspended in $\left(x_{0}, y_{0}\right)$, which is at the center of the active magnetic bearing. The deviation of the rotor between the actual position $(x, y)$ and the set $\left(x_{0}, y_{0}\right)$ is detected by a noncontact position sensor, which is fed back to the control device. The control objective is to maintain the rotor near the expected value $\left(x_{0}, y_{0}\right)$. In this way, according to the deviation between the actual position $(x, y)$ and the set position $\left(x_{0}, y_{0}\right)$, a position adjustment command signal is produced by the controller, which is converted into a current by a power amplifier. The current is inputted to the bearing electromagnet coil to produce the desired electromagnetic control force $\left(F_{m, x}, F_{m, y}\right)$, which makes rotor stability suspension [11].

\section{Dynamic Model of the Flexible Spindle Cutting System Supported by Active Magnetic Bearings}

3.1. Finite Element Model of Magnetic Bearing-Flexible Spindle Cutting System. The simplified structure of the flexible electric spindle with an active magnetic bearing is shown in Figure 2(a). The active magnetic bearing-flexible spindle cutting system mainly includes a high-speed motor rotor, different sizes of elastic spindle sections, active magnetic bearings, cutter holder, cutter, and other components. When the finite element model of the flexible magnetic spindle machining system is established, the following is simplified: the rotor, cutter holder, and cutter of the motor are equivalent to isotropic materials in order to reduce the computer's calculation time. The simplified electric spindle rotor system is divided into shafts of different sizes, magnetic bearings, cutters, and other units along the central axis of the spindle, and the units are connected to each other at the nodes. The finite element model of the spindle rotor system is shown in Figure 2(b). $n 1, n 2, n 3, n 4, n 5, n 6, n 7$, and $n 8$ are nodes in the motorized spindle rotor.

The finite element model of the spindle rotor system is established according to the rotor dynamics theory. Among them, the magnetic bearing characteristics, in the form of electromagnetic force, are applied to the corresponding spindle node. The dynamic cutting process is also a dynamic cutting force, which is applied to the cutter node.

The center axis of the spindle is defined as the $z$-axis, and the plane perpendicular to the $z$-axis is the $X-Y$ plane to establish the OXYZ coordinate system. So, the spindle position can be represented by the coordinates of the $x$ - and $y$-axis and cross section of the deflection angles $\theta_{x}$ and $\theta_{y}$ in any section. The finite element model of the flexible magnetic spindle machining system is $[12,13]$

$$
M_{s} \ddot{U}+\left(D_{s}+\Omega_{s} G_{s}\right) \dot{U}+K_{s} U=F_{\mathrm{am}}+F_{\mathrm{um}}
$$

where $M_{s}$ represents the mass matrix, $K_{s}$ represents the stiffness matrix, $D_{s}$ represents the damping matrix, $G_{s}$ represents the gyro matrix, $\Omega_{s}$ represents the steady-state working speed of the electric spindle, $F_{\text {um }}$ represents the unbalanced force of all the nodes on the electric spindle rotor, $F_{\mathrm{am}}$ is the radial electromagnetic control force of the active magnetic bearing, and $n$ represents the total number of unit nodes. The subscripts $x$ and $y$ represent the direction of the coordinate axes, respectively. The stiffness and damping in (1) mainly refer to the equivalent stiffness and damping generated by the active magnetic bearing and the controller, the stiffness and structural damping of the spindle, and the stiffness and damping in the dynamic cutting process.

\subsection{Equivalent Electromagnetic Force Model of Active Mag-} netic Bearings. The equivalent electromagnetic force model of the active magnetic bearing has been deduced in detail in the relevant books and literature, and the process is not deduced here. This paper still adopts the differential 


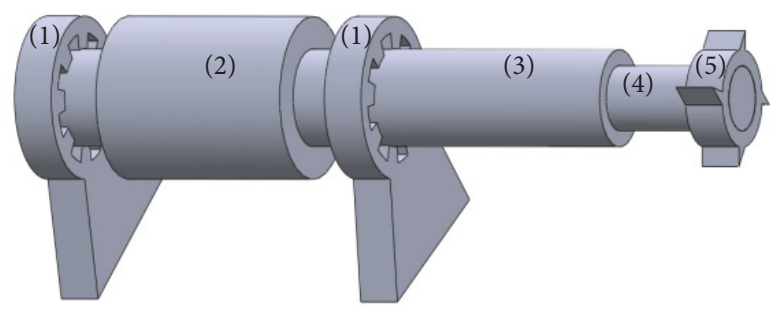

(a) The simplified structure of the flexible motorized spindle with the active magnetic bearing. (1) Active magnetic bearing (AMB), (2) rotor,

(3) motorized spindle, (4) cutter holder, and (5) cutter

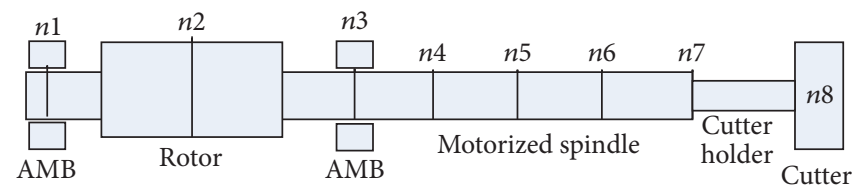

(b) The finite element model of the spindle rotor system

FIgURE 2: The flexible motorized spindle with the active magnetic bearing.

electromagnetic structure to carry on the active electromagnetic force model establishment [14]. The differential control structure of the active magnetic bearing is shown in Figure 3.

The two opposing magnetic poles in the same coordinate axis direction apply electromagnetic force to the electric spindle rotor at the same time, but the bias current $I_{0}$ of the two poles is the same and the control current $\Delta i$ is opposite. Therefore, the equivalent electromagnetic force in the direction of the two opposing C-type magnetic poles is

$$
\begin{aligned}
F_{m, x} & =\frac{4 \mu_{0} A N_{c}^{2} I_{0} \cos \alpha}{\delta_{0}^{2}} \Delta i-\frac{4 \mu_{0} A N_{c}^{2} I_{0}^{2} \cos ^{2} \alpha}{\delta_{0}^{3}} \Delta x \\
& =C_{i} \Delta i+C_{x} \Delta x \\
F_{m, y} & =\frac{4 \mu_{0} A N_{c}^{2} I_{0} \cos \alpha}{\delta_{0}^{2}} \Delta i-\frac{4 \mu_{0} A N_{c}^{2} I_{0}^{2} \cos ^{2} \alpha}{\delta_{0}^{3}} \Delta y \\
& =C_{i} \Delta i+C_{y} \Delta y,
\end{aligned}
$$

where

$$
\begin{aligned}
C_{i} & =\frac{4 \mu_{0} A N_{c}^{2} I_{0} \cos \alpha}{\delta_{0}^{2}} ; \\
C_{x} & =C_{y}=\frac{4 \mu_{0} A N_{c}^{2} I_{0}^{2} \cos ^{2} \alpha}{\delta_{0}^{3}},
\end{aligned}
$$

where $C_{i}$ represents the equivalent current stiffness coefficient of the magnetic bearing and $C_{x}$ and $C_{y}$ represent the equivalent displacement stiffness coefficient in the $x$ direction and $y$-direction of the magnetic bearing. When the active magnetic bearing is running in the linear region near the operating point, the current stiffness coefficient and the displacement stiffness coefficient can be equivalent to a constant.

\section{Unbalanced Vibration Controls for the Multinode Flexible Spindle Rotor System}

4.1. Traditional Influence Coefficient Method. According to the finite element method, when there are the unbalanced masses of em2, em5, em7, and em8 in the $n 2, n 5, n 7$, and $n 8$ nodes of the flexible electric spindle rotor, the differential equation of motion of the flexible electric spindle rotor system is

$$
\begin{aligned}
& M_{s} \ddot{U}+\left(D_{s}+\Omega_{s} G_{s}\right) \dot{U}+K_{s} U
\end{aligned}
$$

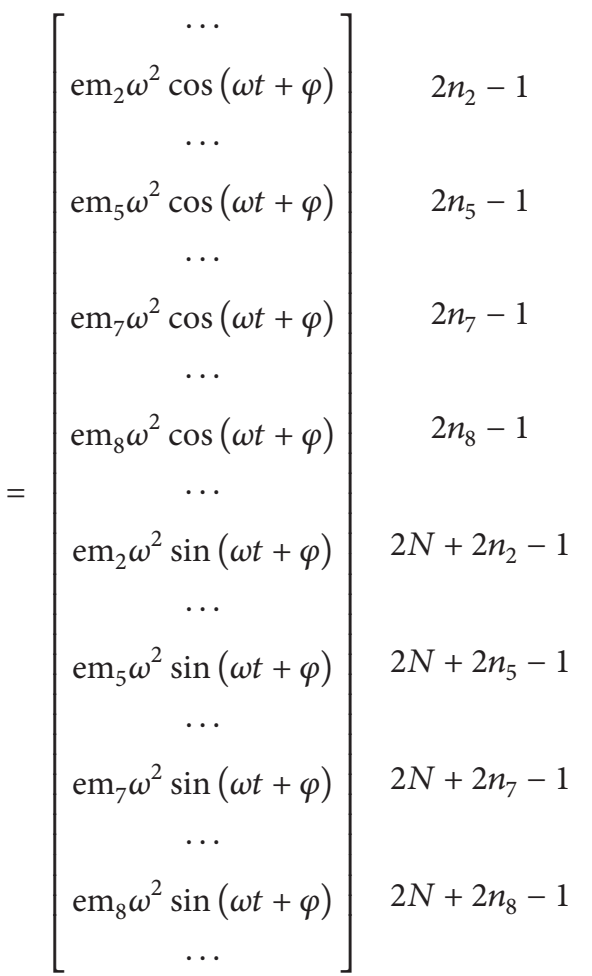

If the flexible electric spindle rotor system is in the linear range, the relationship between response and the external excitation force of each node's own degree can always be expressed as the following [15]:

$$
\begin{aligned}
{\left[\begin{array}{c}
v_{R 1} \\
v_{R 2} \\
\vdots \\
v_{R n}
\end{array}\right] } & =\left[\begin{array}{cccc}
c_{11} & c_{12} & \cdots & c_{1 m} \\
c_{21} & & & \\
\vdots & & \ddots & \vdots \\
c_{n 1} & & \cdots & c_{n m}
\end{array}\right]\left[\begin{array}{c}
f_{c 1} \\
f_{c 2} \\
\vdots \\
f_{c n}
\end{array}\right]+\left[\begin{array}{c}
v_{i 1} \\
v_{i 2} \\
\vdots \\
v_{i n}
\end{array}\right], \\
V_{R} & =C F_{c}+V_{i},
\end{aligned}
$$

where $n$ is the number of nodes. The node to which the correction force is applied is called the excitation node, and $m$ 


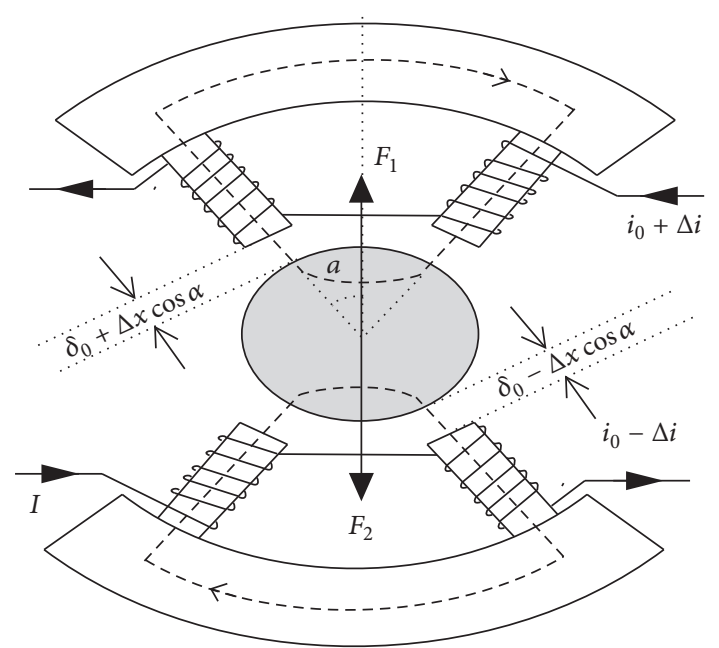

FIGURE 3: Active magnetic bearing differential structure.

is the number of the excitation nodes. $V_{i}$ indicates the original vibration of the node, as the $n \times 1$ dimension vector. $V_{R}$ is the residual vibration of the node after the unbalanced correction force, which is the $n \times 1$ dimension vector. $F_{c}$ represents the unbalanced correction force vector, which is the $m \times 1$ dimension vector. $C$ is known as the coefficient of influence matrix, the $n \times m$ complex matrix, which can be calculated by a theoretical or an experimental test method. The purpose of the flexible spindle vibration control is to suppress the vibration of the unbalanced vibration nodes, that is, make the residual vibration $V_{R}$ be zero vectors or be minimal.

The influence coefficient matrix is composed of an influence coefficient $c_{i j}$, and each influence coefficient indicates that the vibration change detected at the $i$ th node after the $j$ th excitation node is applied to the unit correction force. The influence coefficient reflects the transfer function relationship between the excitation force of the excitation node and the node response at a given speed. In the linear range, the influence coefficient matrix elements are only functions of the speed. Only in the constant speed condition is the influence coefficient matrix the constant matrix.

$c_{i j}$ can be obtained by the following equation:

$$
c_{i j}=\frac{\left(v_{i}\right)_{\mathrm{fin}}-\left(v_{i}\right)_{\mathrm{ini}}}{\left(f_{c, j}\right)_{\mathrm{fin}}-\left(f_{c, j}\right)_{\mathrm{ini}}}=\frac{\Delta v_{i}}{\Delta f_{c, j}},
$$

where the subscript ini represents the initial value before the correction and the subscript fin represents the final value after correction.

Assume that the target node response is $V_{R, k}$ under the effect of $F_{c, k}$; the incentive is changed to $F_{c, k+1}$; the target node response becomes $V_{R, k+1}$. Then,

$$
\begin{gathered}
V_{R, k}=C F_{c, k}+V_{i}, \\
V_{R, k+1}=C F_{c, k+1}+V_{i} .
\end{gathered}
$$

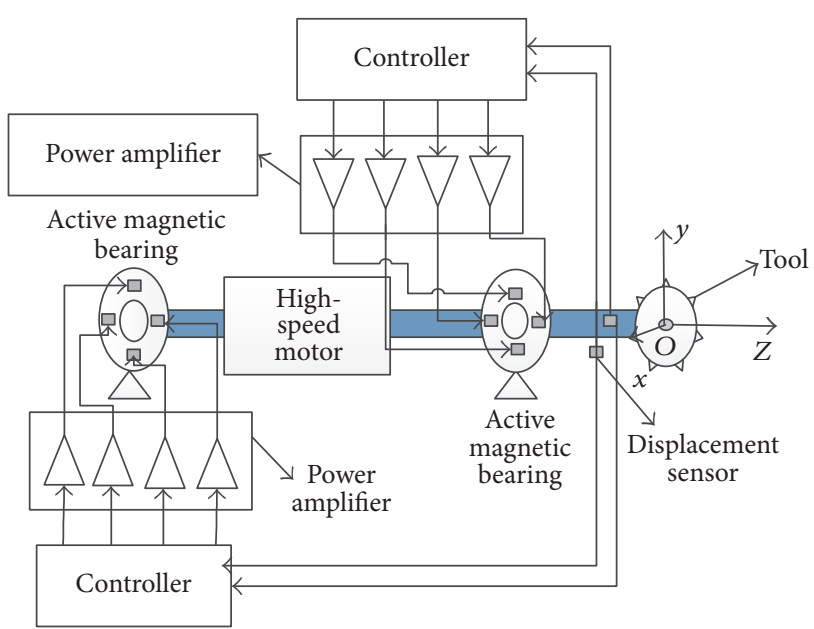

FIGURE 4: Active control for multinode unbalanced vibration in cutting base on active magnetic bearings.

In order for the excitation $F_{c, k+1}$ to completely suppress the vibration of the target node, that is, $V_{R, k+1}=0$, the following can be solved:

$$
F_{c, k+1}=F_{c, k}-C^{-1} V_{R, k} .
$$

Equation (8) shows that if the influence coefficient matrix $C$ is known, the corrected force $F_{c, k+1}$ can be accurately obtained by a single operation to make rotor vibration $V_{R, k+1}$ zero.

Based on the finite element software developed by Matlab, the multinode unbalanced vibration of the flexible electric spindle rotor system supported by the active magnetic bearing is studied. The spindle parameters are the spindle length $l=1430 \mathrm{~mm}$, core length $l_{e}=200 \mathrm{~mm}$, rotor outer diameter $D_{r \text {,out }}=100 \mathrm{~mm}$, and rotor inner diameter $D_{r, \text { in }}=50 \mathrm{~mm}$. Under the active magnetic bearing with the equivalent stiffness of $1 \times 10^{6} \mathrm{~N} / \mathrm{m}$, the unbalance is given as $1 \mathrm{~g} \cdot \mathrm{mm}$, which is applied to rotor nodes $2,5,7$, and 8 . The phases of the unbalance in $n 2, n 5, n 7$, and $n 8$ are $x$-direction, $y$-direction, $-x$-direction, and $-y$-direction, respectively. The control scheme is shown in Figure 4. According to the vibration displacement of the cutter in the cutting process, the equivalent stiffness and equivalent damping of the active magnetic bearing are adjusted in real time to control the multinode unbalanced vibration of the rotating spindle rotor system. The vibration displacement is inputted to the controller (the traditional influence coefficient method) to produce the required control force. The required control force is amplified by the power amplifier and converted to control current required by the active magnetic bearing. And so, the multinode unbalanced vibration of the cutting spindle system in the cutting process is controlled. The influence coefficient is obtained by using the offline measurement in order to eliminate the interference of inaccurate problems, which is obtained by a look-up table in the simulation.

The original vibration amplitudes of electric spindle rotor nodes $1,2,3,5,7$, and 8 in the range of 1 to $500 \mathrm{rad} / \mathrm{s}$ are shown in Figure 5. The rotor has two vibration peaks at critical speed 


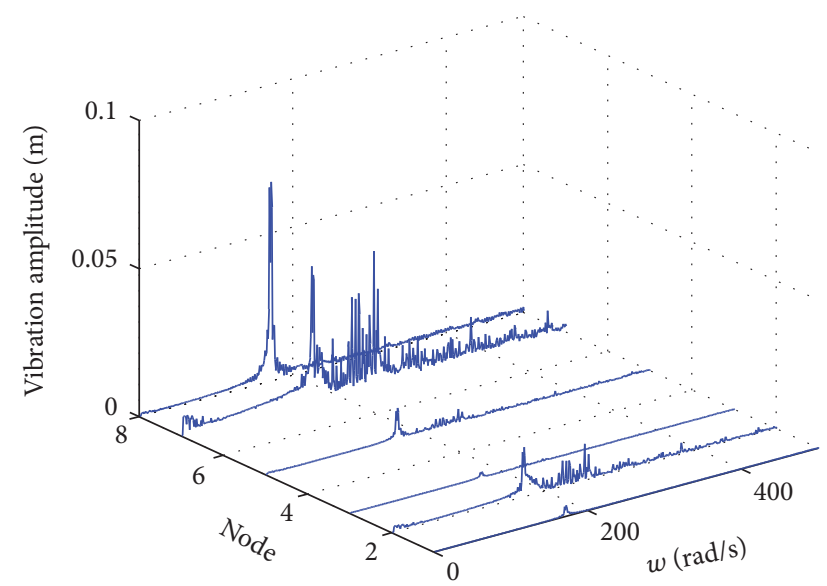

FIGURE 5: Original vibration of 1, 2, 3, 5, 7, and 8 nodes of the rotor.

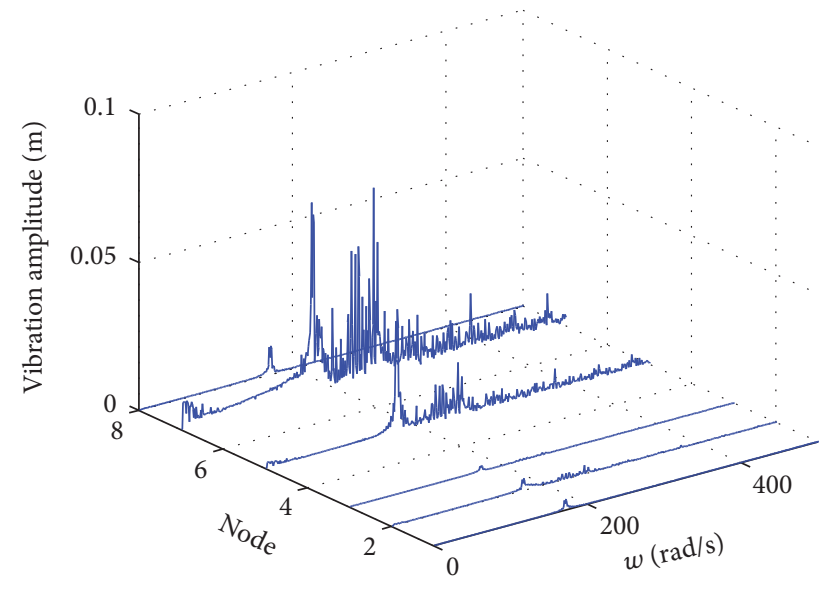

FIGURE 6: Nodes 2 and 8 vibrations controlled.

positions 183.8 and $224.6 \mathrm{rad} / \mathrm{s}$, respectively. Among them, the vibration of the first-order bending critical speed is larger. The vibration amplitudes of nodes $2,5,7$, and 8 are about $25 \mathrm{~mm}, 42 \mathrm{~mm}, 40 \mathrm{~mm}$, and $70 \mathrm{~mm}$.

According to (8), the correcting electromagnetic force required for double nodes vibration controlled with double excitation nodes can be calculated in real time. When the active magnetic bearing nodes 1 and 3 are used as the excitation nodes, the vibration amplitudes of the rotor nodes 2 and 8 are shown in Figure 6. The vibration amplitudes of the rotor nodes 5 and 8 are shown in Figure 7. The vibration amplitudes of the rotor nodes 2 and 8 are shown in Figure 8 .

Figures 6 to 8 show that the vibration control of the specified nodes is very effective, and the amplitude of vibration is reduced greatly. As can be clearly seen from Figures 6 to 8 , since the only control target of vibration control is to eliminate the vibration of the specific nodes, the vibration of the other nodes is not taken into account. In the first-order bending critical speed, only the vibrations of the specific nodes are suppressed, while the other nonspecific nodes vibrations are aggravated by different degrees.

At this point, the real-time vibration control for the flexible motor spindle rotor system in the entire speed range is

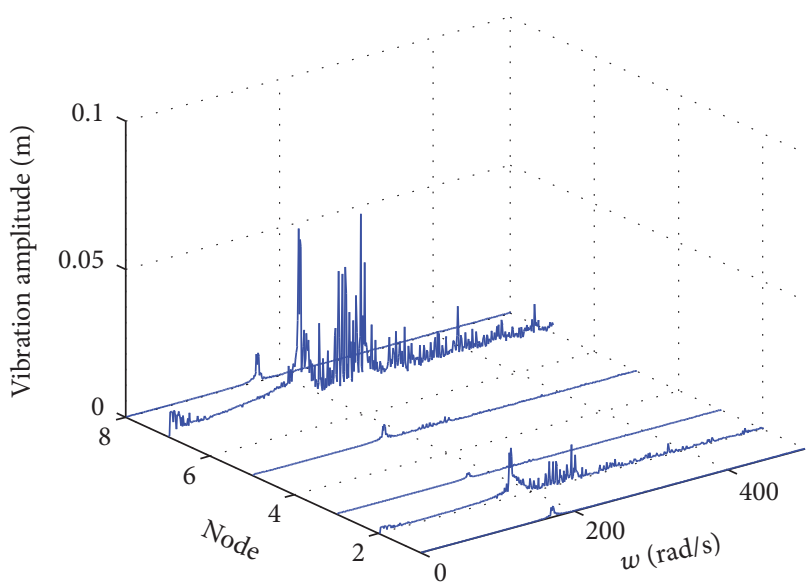

FIGURE 7: Nodes 5 and 8 vibrations controlled.

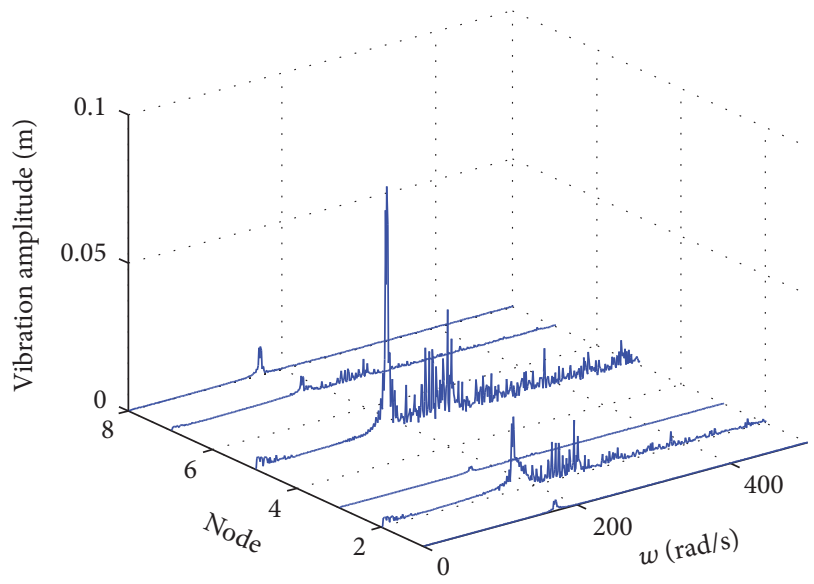

Figure 8: Nodes 7 and 8 vibrations controlled.

needed to consider the following issues: in some known speed points, such as critical speed, the applied electromagnetic force may cause excessive vibration on other nonspecified nodes by the above traditional influence coefficient control method to simply eliminate the vibration of the specified node.

The solution to the above problem is to require the vibration control method for multinode unbalanced vibrations of the flexible spindle rotor system which has the ability to take into account multinode unbalanced vibrations and even control the vibration of all nodes in the rotor system at the same time.

4.2. Weighted Optimization of the Influence Coefficient Method. Equation (5) is a multivariate system. When the number of unbalanced vibration nodes and the number of stimulating nodes are equal $(n=m)$, the vibrations of all nodes are corrected to zero by a single solution of unbalanced correction force vectors. When $n<m$, there is a set of solutions for completely suppressing the unbalanced vibration nodes. When $n>m$, all the unbalanced vibration nodes cannot be corrected to zero at the same time. In general, the rotor is usually supported by two active magnetic 
bearings $(m=2)$. According to the above analysis, only the two unbalanced vibration nodes can be precisely controlled by two active magnetic bearings.

On the other hand, the correction force for the vibration control of the flexible spindle rotor system can be calculated by (8).

In the case of $n \neq m$, since $C$ is not a square matrix, its inverse matrix does not exist, and the correction force of (8) cannot be used. In fact, when $n>m$, (5) cannot be solved by the multivariate system. This leads to the problem that the number of unbalanced vibration nodes cannot be more than the number of the excitations in vibration controlling for the flexible electric spindle rotor. A method of suppressing the vibration of the flexible rotor is proposed [15], which can avoid the nonsquare matrix inversion problem in the multivariate system. The unbalanced vibrations of all nodes in the whole rotor can be used as the objective function to realize the optimal control.

In the evaluation of the vibration of the flexible spindle rotor system, it is not equally important for vibration of all the nodes. In order to reasonably weigh the vibration of each node position, you can determine the evaluation of the vibration of each node position. The vibration evaluation weight matrix $W$ is defined as an $n \times n$ diagonal matrix:

$$
W=\left[\begin{array}{cccc}
w_{1} & & & 0 \\
& w_{2} & & \\
& & \ddots & \\
0 & & & w_{n}
\end{array}\right] .
$$

The element $w_{i}$ in the weight matrix $W$ defines the weight of the vibration at the $i$ th node position.

Weighted square and minimization of vibration amplitude is the objective function, which is the most commonly used in optimal control of rotor vibration; that is,

$$
J=\frac{1}{2} V_{R, k}{ }^{*} W V_{R, k}
$$

where $J$ is the instantaneous value of the objective function. The superscript $*$ represents the Hermitian transform of the matrix. According to (10), the optimal control problem of rotor vibration suppression becomes a problem of how to minimize the objective function $J$.

Assuming that the original unbalanced vibration $V_{i}$ remains constant during being controlled, the current rotor vibration can be expressed by the amount of change of rotor vibration and correction force for the previous iteration period; that is,

$$
V_{R, k+1}=C\left(F_{c, k+1}-F_{c, k}\right)+V_{R, k}
$$

Substituting (9)-(10) into (11), we can get the relationship between the objective function $J$, the rotor vibration, and the correction force of the last iteration period:

$$
\begin{aligned}
J= & \frac{1}{2}\left(C\left(F_{c, k+1}-F_{c, k}\right)+V_{R, k}\right)^{*} \\
& \cdot W\left(C\left(F_{c, k+1}-F_{c, k}\right)+V_{R, k}\right),
\end{aligned}
$$

where $C, F_{c, k}$, and $V_{R, k}$ are known in (12). In order to make the objective function $J$ the minimum extremum by the correction force at time $k+1$, it can be obtained by the partial derivative of $J$; that is,

$$
\frac{\partial J}{\partial F_{c, k+1}}=0
$$

It is necessary that the quadratic function of $J$ is $F_{c, k+1}$ to ensure the minimum value of the partial derivative of the objective function $J$. The condition is that the vibration evaluation weight matrix $W$ is positive definite matrix and must be full rank. In order to make the influence factor matrix satisfy the full-rank condition, according to the literature [15], the selected active magnetic bearing excitation position follows two principles:

(1) Avoid setting the active magnetic bearing excitation position on the node of the vibration mode.

(2) Make sure that the effect of the correction force from each excitation node on the vibration vectors of the rotor system is independent; that is, the correction forces of each excitation node are not coupled to each other.

The partial derivation of $J$ is obtained:

$$
\frac{\partial J}{\partial F_{c, k+1}}=C^{*} W\left(C\left(F_{c, k+1}-F_{c, k}\right)+V_{R, k}\right) .
$$

According to (13) and (14), the minimum value $F_{c, \text { min }}$ of the objective function $J$ can be obtained:

$$
\begin{aligned}
F_{c, \min } & =\left[C^{*} W C\right]^{-1}\left[C^{*} W C F_{c, k}-C^{*} W V_{R, k}\right] \\
& =\left[C^{*} W C\right]^{-1}\left[C^{*} W\left[C F_{c, k}-V_{R, k}\right]\right] \\
& =-\left[C^{*} W C\right]^{-1} C^{*} W V_{i} .
\end{aligned}
$$

Of course, because the actual $C$ and $V_{i}$ are unknown, $F_{c, \text { min }}$ can gradually approach the estimate of the influence coefficient and the robustness of the iterative convergence, as follows:

$$
F_{c, k+1}=\left[C^{*} W C\right]^{-1}\left[C^{*} W\left[C F_{c, k}-V_{R, k}\right]\right] .
$$

We can see that (16) is exactly equivalent to (8) if $C$ is square matrix and $W$ is unit matrix. The greatest contribution of (16) is to avoid the direct inversion problem when the coefficient matrix is not a square matrix. Equation (16) can control the vibrations of an unlimited number of nodes according to the rules of weights.

When the objective function $J$ is the minimum extreme value, the residual vibrations of the rotor nodes can be solved by substituting (16) into (8):

$$
\begin{aligned}
V_{R, \min } & =C\left[-\left[C^{*} W C\right]^{-1} C^{*} W V_{i}\right]+V_{i} \\
& =\left[I_{n \times n}-C\left[C^{*} W C\right]^{-1} C^{*} W\right] V_{i},
\end{aligned}
$$

where $I_{n \times n}$ represents the $n \times n$ unit matrix. 


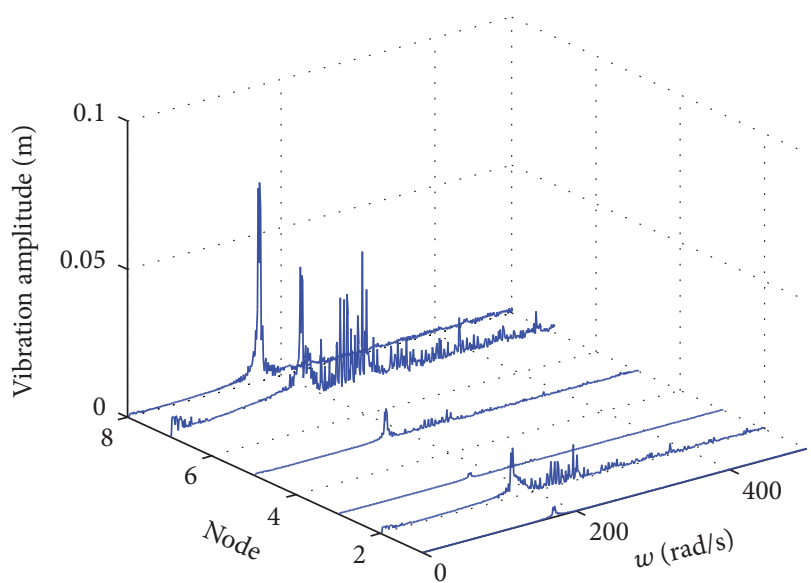

(a) Vibration of nodes without being controlled

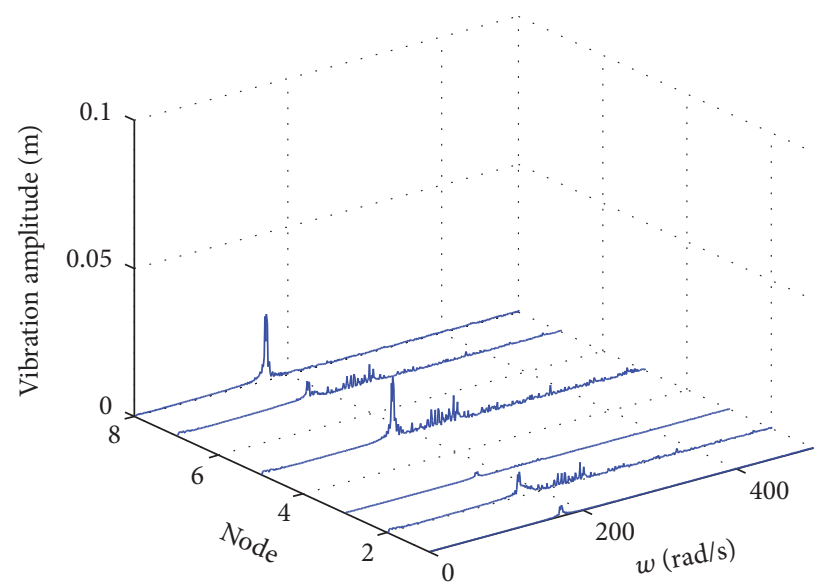

(b) Vibration of nodes with control $\left(W_{1}\right)$

Figure 9: Vibration weighted optimal control effect of nodes 1, 2, 3, 5, 7, and 8.

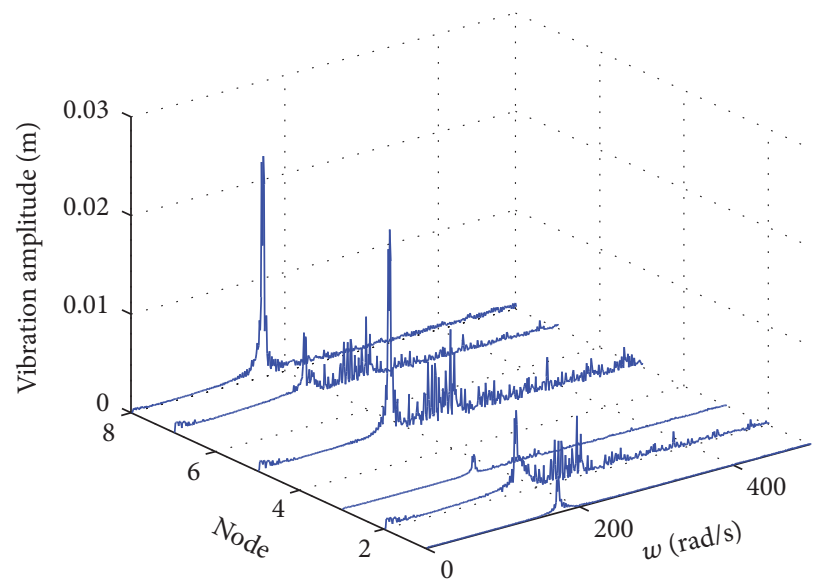

(a) Vibration of nodes controlled $(W)$

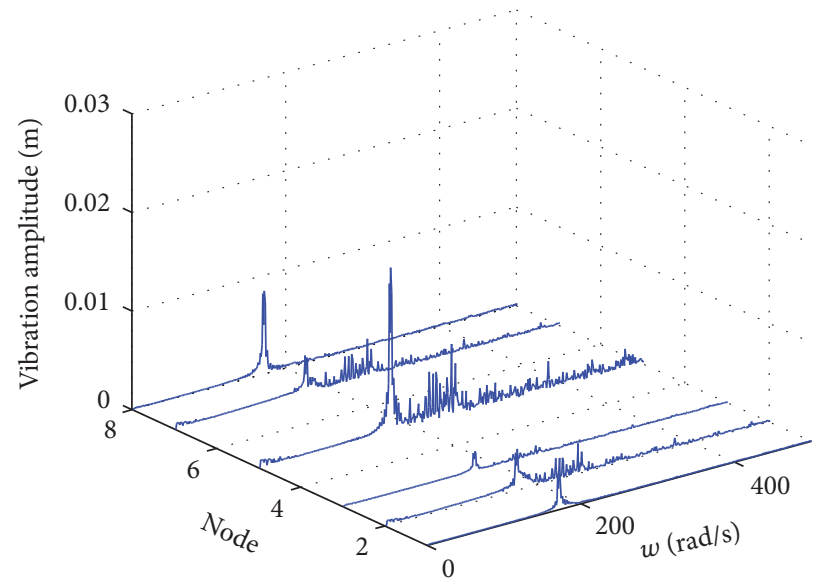

(b) Vibration of nodes controlled ( $w_{2}$ and $w_{8}$ are adjusted)

FIGURE 10: Weighted optimal control for nodes 2 and 8 vibration by adjusting weights $w_{2}$ and $w_{8}$.

In order to verify the control effect of the vibration of multinode unbalanced vibration by using weighted optimization of the influence coefficient method, the simulation is carried out in Matlab. The control scheme is the same as the one in Figure 4. The controller is replaced with weighted optimization of the influence coefficient method. The influence coefficient matrix of the rotor is also determined by the offline method, which is obtained by looking up the table according to the rotor speed in real-time control. In simulation, the active magnetic bearing nodes 1 and 3 are taken as the excitation nodes, and nodes 2, 5, 7, and 8 are taken as the unbalanced vibration nodes. All the vibration weights of nodes are equal; that is, $W$ is the unit matrix. Figure 9 shows the vibration control effect in the range of 1 to $500 \mathrm{rad} / \mathrm{s}$. When the weights $w_{2}$ and $w_{8}$ in $W$ are changed, the control effects of nodes 2, 5, 7, and 8 are shown in Figure 10. As a comparison, Figure 11 shows the comparison of the vibration response of node 2 with the different weight. Figure 12 shows the comparison of the vibration response of node 5 with the different weight. Figure 13 shows the comparison of the vibration response of node 7 with the different weight. Figure 14 shows the comparison of the vibration response of node 8 with the different weight. In Figures 11 to 14, the blue solid line is the vibration control of nodes $2,5,7$, and 8 with the unit matrix $W$, and the red dotted line is the vibration control effect of nodes 2 and 8 after the adjusted weights $w_{2}$ and $w_{8}$ in $W$ to pursue the perfect minimization vibrations of nodes 2 and 8.

As can be seen from Figures 11 to 14, the vibration of nodes 2 and 8 is lower than the previous one by the adjusted weight, but there is little suppression for the vibration of node 5 and node 7. As long as the appropriate weight is selected, you can control unbalanced vibrations of all the nodes on the spindle rotor.

It can be seen that, under the control strategy of multinode weighted vibration objective function $J$, it is very effective to reduce the multinode unbalanced vibration of the flexible spindle rotor system. When weights are adjusted, some nodes have better control. So, the right weight $W$ will directly determine the vibration control effect of multiple unbalanced 


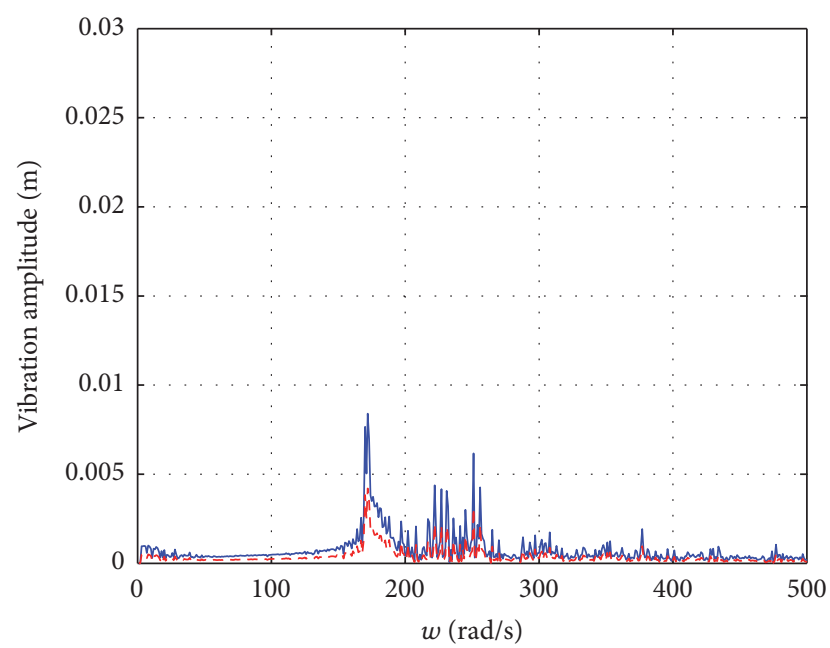

FIGURE 11: Comparison of two control effects (node 2).

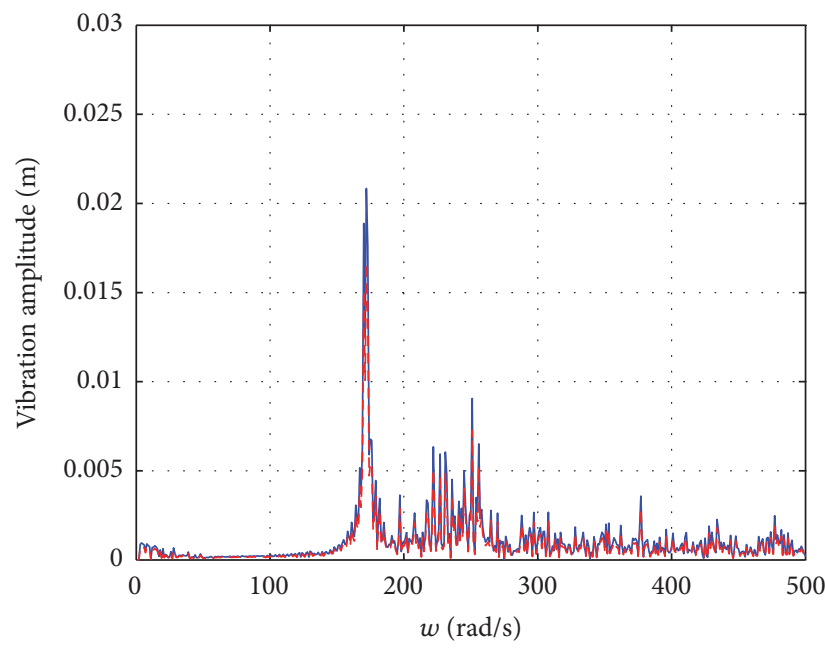

FiguRE 12: Comparison of two control effects (node 5).

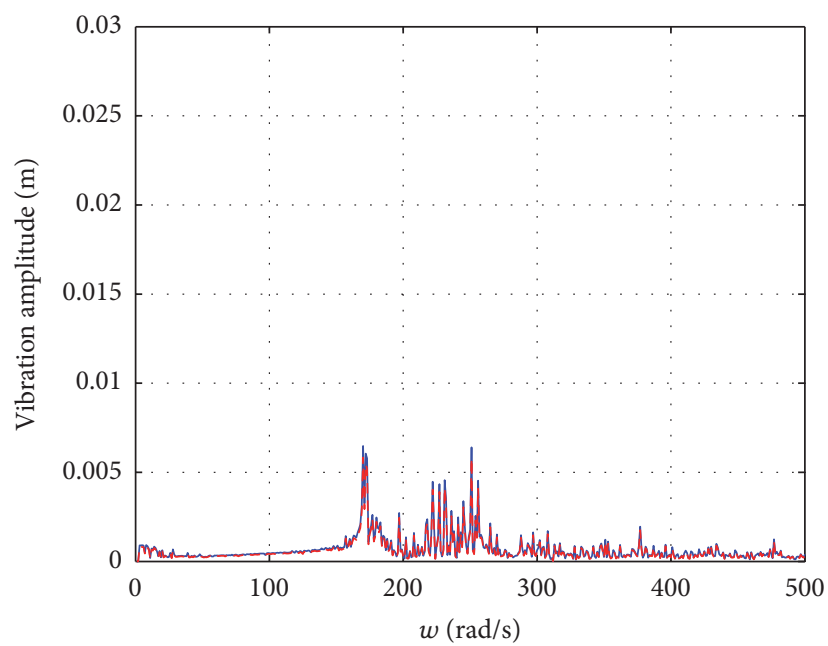

FIgURE 13: Comparison of two control effects (node 7).

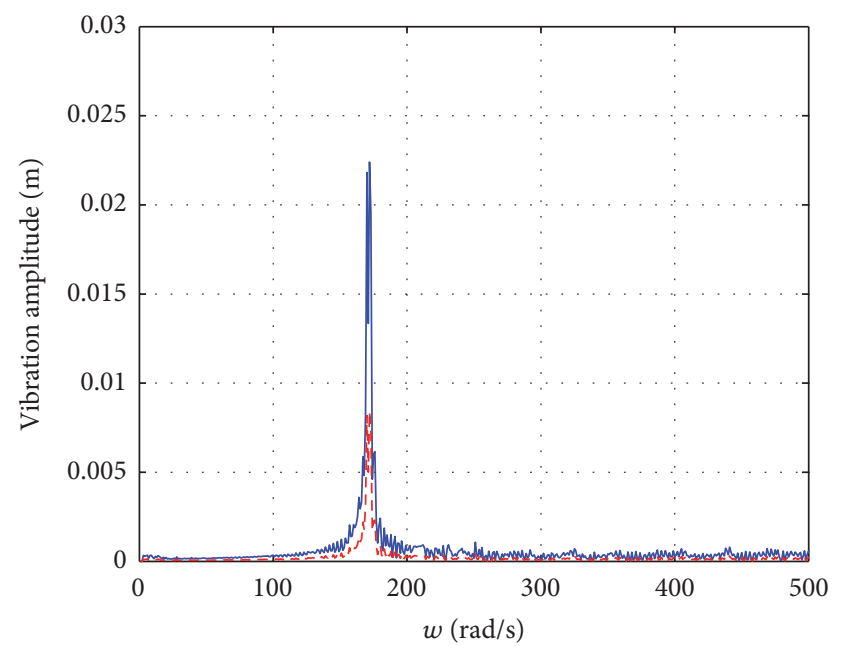

FIGURE 14: Comparison of two control effects (node 8).

vibration nodes on the whole rotor. In particular, the multinode weighted objective function $J$ takes into account the vibration of each node and does not cause the vibration peak at other unknown nodes.

\section{Conclusions}

It is necessary to solve the multivariate equations to calculate the required electromagnetic force based on the traditional influence coefficient method for the vibration suppression of the flexible electric spindle rotor, which led to the number of vibration suppression nodes being not more than the number of excitation nodes of the active magnetic bearing. In order to avoid solving the nonsquare matrix inverse problem of multivariate equations, the optimal influence coefficient control method with weights for multinode unbalanced vibration of flexible electric spindle rotors is proposed on the basis of the traditional influence coefficient method, which can use all the unbalanced vibration of nodes as the objective function to achieve the optimal control, and the control effect is proved by the comparison simulation. The simulation results show that the optimal control method of the flexible electric spindle rotor system with mixed weight objective function has a good effect of suppressing the multinode unbalanced vibration of the rotor system.

\section{Conflicts of Interest}

The authors declare that they have no conflicts of interest regarding the publication of this paper.

\section{Acknowledgments}

This research was supported by the National Natural Science Foundation of China (51505296). 


\section{References}

[1] Y. Zhong, Y. Z. He, and Z. Wang, Rotor Dynamics, Tsinghua University Press, 1987.

[2] C.-Y. Tseng, T.-W. Shih, and J.-T. Lin, "A Kalman filter-based automatic rotor dynamic balancing scheme for electric motor mass production," Materials Science Forum, vol. 505-507, no. 2, pp. 997-1002, 2006.

[3] H. W. Li, Y. Xu, and H. D. Gu, "Field dynamic balancing method in AMB flexible rotor system," China Mechanical Engineering, vol. 19, no. 12, pp. 1419-1428, 2008.

[4] C.-S. Kim and C.-W. Lee, "In situ runout identification in active magnetic bearing system by extended influence coefficient method," IEEE/ASME Transactions on Mechatronics, vol. 2, no. 1, pp. 51-57, 1997.

[5] Y. Kang, T. Lin, and M. Chu, "Design and simulation of a neuralPD controller for automatic balancing of rotor," in Proceedings of the 3rd International Symposium on Neural Networks, pp. 11041109, Chengdu, China, 2006.

[6] C. D. Untaroiu, P. E. Allaire, and W. C. Foiles, "Balancing of flexible rotors using convex optimization techniques: optimum min-max LMI influence coefficient balancing," Journal of Vibration and Acoustics, vol. 130, no. 2, Article ID 021006, 2008.

[7] Z. Yu, L. T. Meng, and L. M. King, "Electromagnetic bearing actuator for active vibration control of a flexible rotor," Proceedings of the Institution of Mechanical Engineers Part C, vol. 212, no. 8, pp. 705-716, 1998.

[8] C. Knospe, "Robustness of adaptive unbalance control of rotors with magnetic bearings," Journal of Vibration and Control, vol. 2, no. 1, pp. 33-52, 1996.

[9] S.-H. Lee, B.-S. Kim, J.-D. Moon et al., "A study on active balancing for rotating machinery using influence coefficient method," in Proceedings of the IEEE International Symposium on Computational Intelligence in Robotics and Automation (CIRA '05), pp. 659-664, IEEE, Espoo, Finland, June 2005.

[10] Y. Kang, T.-W. Lin, Y.-J. Chang, Y.-P. Chang, and C.-C. Wang, "Optimal balancing of flexible rotors by minimizing the condition number of influence coefficients," Mechanism and Machine Theory, vol. 43, no. 7, pp. 891-908, 2008.

[11] G. Schweitzer, Eric, and H. Maslen, Magnetic Bearings: Theory, Design And Application to Rotating Machinery, China machine press, 2012.

[12] X. Qiao and C. Zhu, "Active control of milling chatter based on the built-in force actuator," Journal of Mechanical Engineering, vol. 48, no. 1, pp. 185-192, 2012.

[13] X. L. Qiao and C. S. Zhu, "The active vibration attenuation of a built-in motorized milling spindle," Journal of Vibration and Control, vol. 20, no. 13, pp. 1934-1945, 2014.

[14] X. L. Qiao, C. S. Zhu, and Z. X. Zhong, "Stability and control for chatter in cutting based on active magnetic bearings," China Mechanical Engineering, vol. 12, no. 27, pp. 1632-1637, 2016.

[15] W. Stephen, Adaptive optimal control of active balancing systems for high-speed rotating machinery [Ph.D. thesis], The University of Michigan, Ann Arbor, Mich, USA, 1999. 


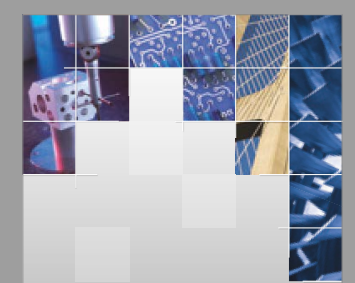

\section{Enfincering}
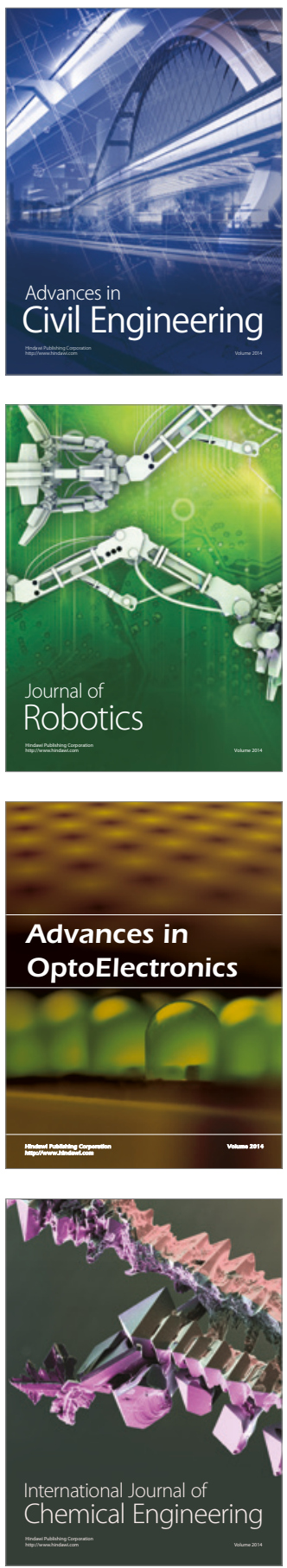

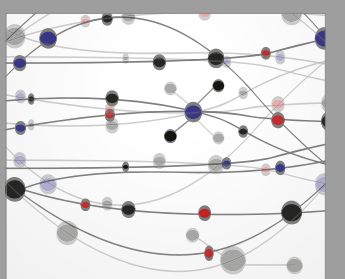

The Scientific World Journal

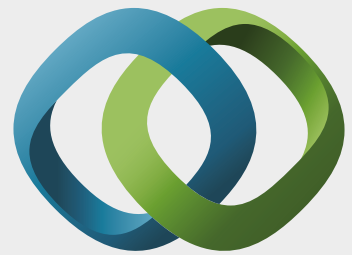

\section{Hindawi}

Submit your manuscripts at

https://www.hindawi.com
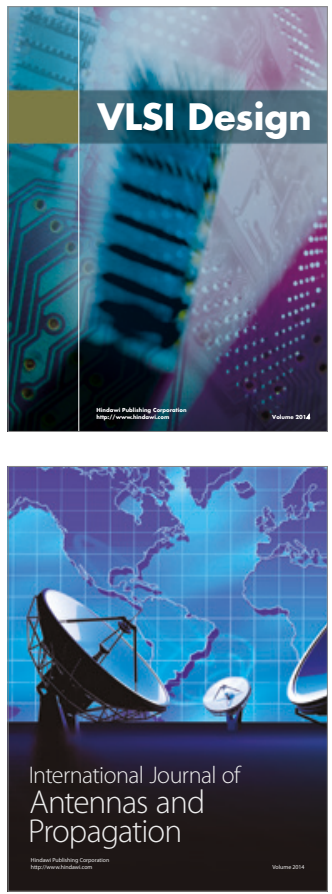

\section{Rotating}

Machinery
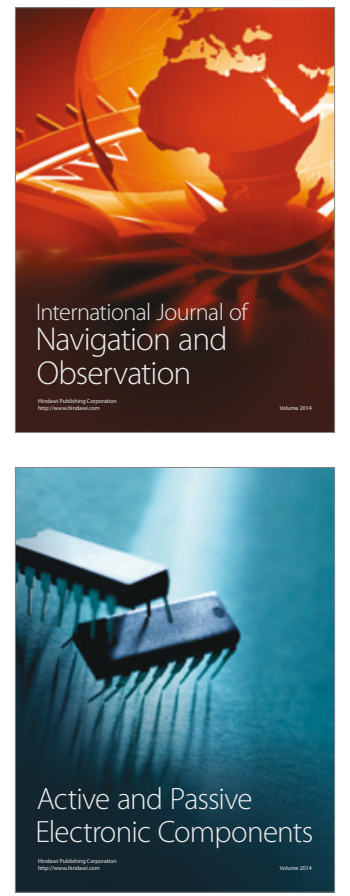
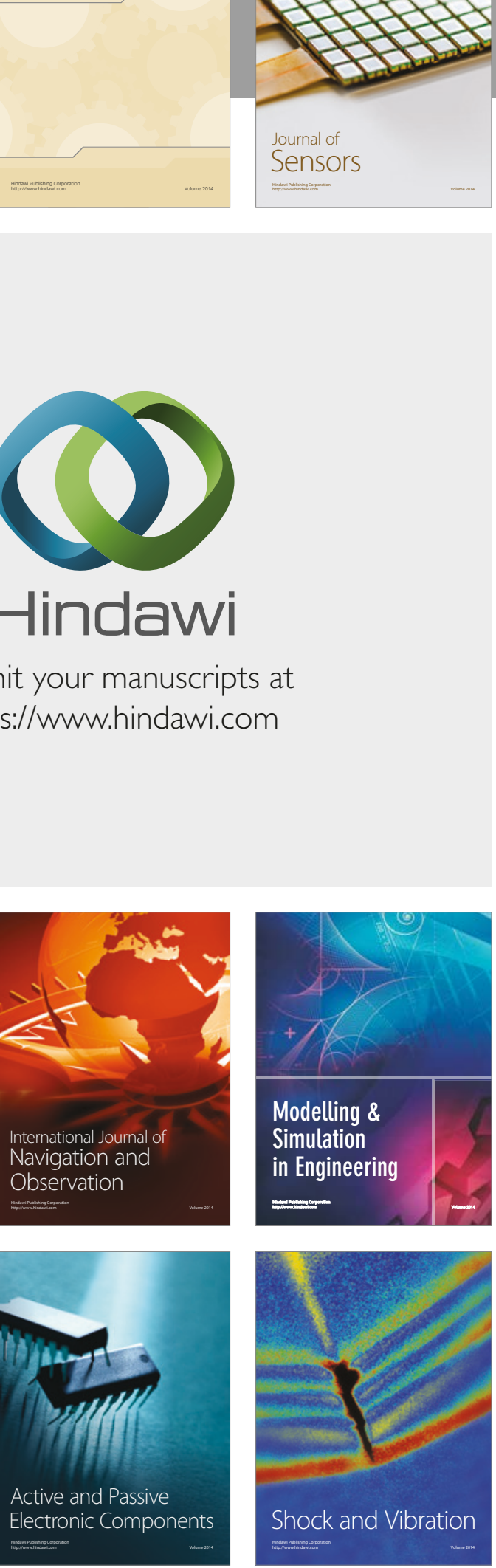
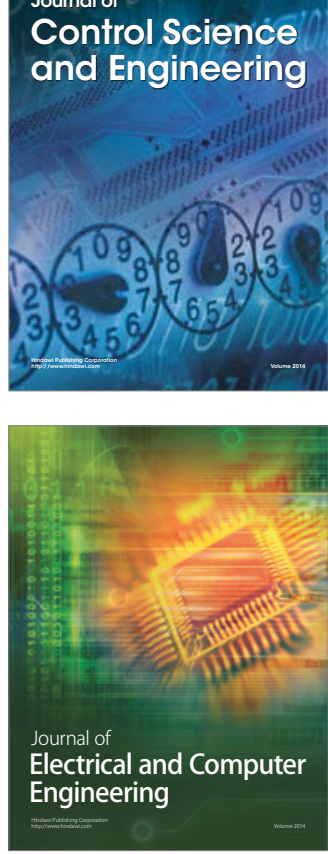

Distributed

Journal of

Control Science

and Engineering
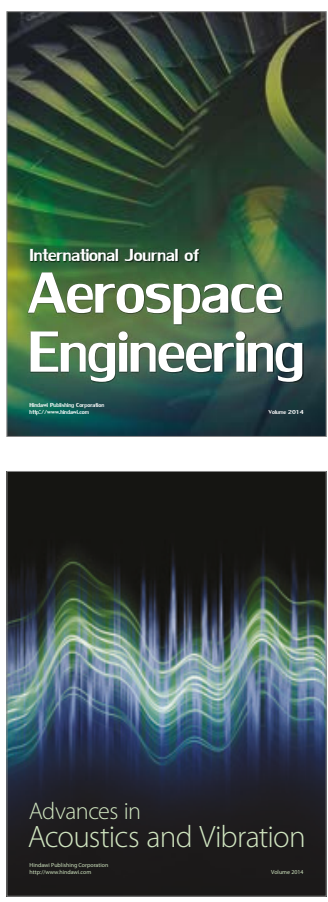

Sensor Networks 